\title{
Dielectric response of a nondegenerate electron gas in semiconductor nanocrystallites
}

\author{
E. van Faassen \\ Debye Institute, Section Interface Physics, Ornstein Laboratory, Utrecht University, 3508 TA, Utrecht, The Netherlands
}

(Received 15 July 1998)

\begin{abstract}
We investigate the low-frequency dielectric response of a dilute electron gas in a small spherical semiconductor particle. The flow of the electrons is described by hydrodynamic equations which incorporate the electrostatic interactions between the electrons in a self-consistent fashion. In the low-frequency regime, the dielectric loss is small and proportional to the frequency, despite substantial field penetration into the semiconductor. The loss remains small even for high doping levels due to effective cancellation between fieldinduced drift and diffusion. The model is used to estimate the complex dielectric constant of a system of weakly conducting nanosized semiconductor particles. The most prominent manifestation of spatial dispersion is that photoinduced changes in the real and imaginary parts of the dielectric constant are positive and of comparable magnitude. [S0163-1829(98)05548-9]
\end{abstract}

\section{INTRODUCTION}

The dynamical properties of mobile charge carriers inside nanostructured semiconductors have been the subject of intensive research. Many interesting phenomena have been found to result from the inhomogeneity on a microscopic scale. ${ }^{1-4}$ Three aspects in particular have attracted attention. First, size quantization effects ${ }^{5-8}$ discretize and modify the electronic states when the typical size of the semiconductor structures becomes of the order of the exciton radius $(\sim 1-5 \mathrm{~nm}){ }^{9,10}$

Second, the high surface-to-volume ratio means that the surface plays a crucial role in the dynamics of charge carriers inside the semiconductor. A technologically important example is given by the very high photoelectron quantum yield found in nanostructured photovoltaic devices. ${ }^{11,12}$ Third, the details of the semiconductor topology (such as connectivity) or the presence of microscopic grain boundaries profoundly affect the macroscopic transport properties of mobile charge carriers. $^{13,14}$ It is a wholly classical phenomenon arising when the structural dimensions are on the same scale as the inhomogeneity in the electron distribution. A further complication arises from possible quantum size effects on charge carrier mobility. ${ }^{15}$

The calculation of dynamical properties such as dielectric response or dynamical screening poses a complicated problem caused by the possibility of buildup of charge on internal surfaces or grain boundaries. Conventionally, the effect of mobile charge carriers on the bulk effective complex dielectric constant is accounted for by adding an imaginary conductivity term to the dielectric constant $\epsilon$ of the medium in which the charge carriers move. For a conductive medium, the Drude model for the complex dielectric constant $\epsilon_{d}=\epsilon_{d}^{\prime}$ $+i \epsilon_{d}^{\prime \prime}$ gives (cf., e.g., Ref. 16)

$$
\epsilon_{d}=\epsilon\left(1+i \frac{\Omega_{p}^{2} \tau}{\omega(1-i \omega \tau)}\right)
$$

Here $\omega$ is the frequency of the applied field, $\tau$ is the momentum relaxation time of the Drude electrons, and $\Omega_{p}$ denotes the plasma frequency inside the medium.
The same expression is often applied to the dielectric properties of a dilute (nondegenerate) electron gas in a semiconducting medium as well. In the regime of low frequencies $(\omega \tau \ll 1)$, this Drude formula predicts that an infinitesimal increase in electron density should induce an increase in dielectric loss $\left(\delta \epsilon_{d}^{\prime \prime}>0\right)$ as well as a much smaller decrease in dielectric response $\left(\delta \epsilon_{d}^{\prime}<0\right)$,

$$
\delta \epsilon_{d}^{\prime}=-\omega \tau \delta \epsilon_{d}^{\prime \prime} \quad(\text { Drude })
$$

In contrast, experimental observation of the photodielectric response of doped porous semiconductors shows that the two are of comparable magnitude and both have a positive sign.

On a more quantitative basis, the Drude formula fails as well: For typical moderately doped semiconductors, conductivities are of the order of $\sigma=1-10(\Omega \mathrm{s})^{-1}$. Therefore, Drude theory predicts that the imaginary part dominates by several orders of magnitude for all submicrowave frequencies $(\omega / 2 \pi \leqslant 10 \mathrm{GHz})$, again in clear conflict with experimental findings of low dielectric loss in doped nanocrystalline powders or suspensions. The reason cannot be excessive screening of the electric fields (as in metals) since the typical Debye screening length is of the order of the crystallite size. This implies substantial penetration of the electric fields into the conducting core of the crystallite.

We therefore consider the dielectric response of a nanosized $n$-doped semiconductor particle. We assume complete ionization of the donor states, which provide a uniform positive space-charge density in which the electrons move. The collective response of the electron gas is described by hydrodynamical flow subjected to an external harmonic field. Solution of the hydrodynamical equations allows us to determine the polarizability and loss factor of the particle, as well as study the degree of screening on a microscopic scale. Subsequently, the results for a single weakly conducting nanocrystallite are used to predict the effective complex dielectric constant of a powder. Serving as a model for the low-frequency dielectric properties of a porous semiconductor, we find good estimates of the dielectric loss factor. In 
particular, the magnitude and sign of the dielectric response find a natural explanation as manifestations of spatial dispersion.

\section{THE HYDRODYNAMICAL MODEL}

The calculation of the dielectric loss in an electronic material is considerably more involved than the treatment of electronic polarization. For the electronic polarization, only the static properties of the ground state need to be considered under weak perturbation by an external static field. The ground-state properties of an electron gas suffice here. In contrast, the dielectric loss is inseparable from the time dependence of the external field, and the dynamic response of the electron gas must be treated in detail. The most detailed and sophisticated theoretical approach is the time-dependent Hartree-Fock (TDHF) method. While conceptually clear, TDHF involves prohibitive computational effort when applied to systems with many electrons. To circumvent this problem, several semiclassical approaches have been proposed which involve only the local electron density as a dynamic variable.

The earliest density method was the Thomas-Fermi theory ${ }^{17}$ developed for the ground-state properties of a highly degenerate electron gas in metals. This Thomas-Fermi formalism provided the theoretical basis for the hydrodynamical Bloch model, ${ }^{18}$ which describes the dynamics of the electronic motion as the flow of a nonviscous compressible fluid. The model has been applied to a wide range of problems involving the degenerate electron liquid in bulk metals and metal clusters. ${ }^{19-22}$ The validity of such semiclassical approaches hinges on the neglect of the discreteness of the underlying ionic lattice and the use of the local electron density. The validation of the hydrodynamic approach down to very small $(N \approx 100)$ metallic clusters has recently been given in Ref. 23 .

For applications considering the motion of charge carriers in a semiconductor particle, the hydrodynamic equations have to be modified to accommodate a low degree of degeneracy and account for momentum loss from the electron flow caused by the effective friction with the semiconductor background. The internal friction (viscosity) of the electron liquid is assumed negligible. In the presence of a local electric field $\mathbf{E}$, the local velocity field $\mathbf{v}(\mathbf{r}, t)$ is related to the local pressure $p$ of the electron gas via the Euler equation

$$
\frac{\partial}{\partial t} \mathbf{v}-(\mathbf{v} \cdot \boldsymbol{\nabla}) \mathbf{v}=-\frac{1}{\tau} \mathbf{v}-\frac{1}{m_{e} n} \boldsymbol{\nabla} p-\frac{e}{m_{e}} \mathbf{E} .
$$

The electron density $n(\mathbf{r}, t)$ and the velocity field are related through the continuity equation

$$
\frac{\partial}{\partial t} n+\operatorname{div}(n \mathbf{v})=0
$$

where $m_{e}$ is the effective electron mass inside the semiconductor. The momentum relaxation time $\tau$ accounts for the momentum loss due to collisions with impurities, lattice imperfections, or phonons. The second term of the Euler equation accounts for diffusive flow induced by density variations, and the final term accounts for the flow induced by the interaction with the local electric field. The electric field is the sum of the external field and the internal fields due to the charge density of the electron gas and of the uniform doping density $n_{0}$ (assuming the donor states to be fully ionized),

$$
\operatorname{div} \mathbf{E}=-\frac{e}{\epsilon \epsilon_{0}}\left(n-n_{0}\right),
$$

where $\epsilon$ is the dielectric constant of the semiconductor and $\epsilon_{0}$ is the permittivity of vacuum.

These equations are supplemented by the equation of state which relates the local pressure $p$ to the local electron density. For nondegenerate semiconductors (low doping density), we assume that the local pressure is given by the equation of state for an ideal classical gas $p(\mathbf{r}, t)=n(\mathbf{r}, t) k T$. For small perturbing fields and small density deviations $\hat{n}(r)$ $=n(\mathbf{r})-n_{0}$, the hydrodynamical equations may be linearized in $\hat{n}$ and take the form of a homogeneous damped wave equation,

$$
-\frac{1}{\Omega_{p}^{2}} \frac{\partial^{2}}{\partial t^{2}} \hat{n}-\tau_{d} \frac{\partial}{\partial t} \hat{n}=\hat{n}-L^{2} \Delta \hat{n},
$$

where $\Omega_{p}=\sqrt{n_{0} e^{2} / \epsilon \epsilon_{0} m_{e}}$ and $L=\sqrt{\epsilon \epsilon_{0} k T / n_{0} e^{2}}$ are the plasma frequency and Debye screening length, respectively. The dielectric relaxation time is given by $\tau_{d}=\left(\Omega_{p}^{2} \tau\right)^{-1}$. For fields with harmonic time dependence $e^{-i \omega t}$, this partial differential equation reduces to the Helmholtz equation with complex Helmholtz length $\zeta$,

$$
\begin{gathered}
\hat{n}-\zeta^{2} \Delta \hat{n}=0, \\
\zeta^{2}=\frac{L^{2}}{1-\frac{\omega^{2}}{\Omega_{p}^{2}}-i \omega \tau_{d}} .
\end{gathered}
$$

In this form, the problem of dielectric response has three characteristic time scales (field frequency $\omega$, plasma frequency $\Omega_{p}$, and dielectric relaxation time $\tau_{d}$ ) and two length scales (particle size and Debye screening length). Supplemented with proper boundary conditions, the Helmholtz equation describes surface plasmons $\left(\omega<\Omega_{p}\right)$ as well as bulk plasmons $\left(\omega>\Omega_{p}\right)$. These collective excitations of the electron liquid appear damped with the characteristic dielectric relaxation time $\tau_{d}$ due to momentum relaxation associated with the hydrodynamic flow. For subgigahertz frequencies and in a typical moderately doped semiconductor, $\Omega_{p}$ $\gg \tau_{d}^{-1} \gg \omega$.

The hydrodynamic equations may be recast in terms of the electrostatic potential $\Phi_{i}$ inside the semiconductor particle [cf. Eq. (4)],

$$
\left(1-\zeta^{2} \Delta\right) \Delta \Phi_{i}=0 .
$$

Outside of the particle, the potential $\Phi_{o}$ satisfies the Poisson equation

$$
\Delta \Phi_{o}=0 .
$$

These equations may be solved in closed form after applying the usual electrostatic boundary conditions at the surface of the particle ( $\operatorname{div} \mathbf{D}=0$ and continuity of the electrostatic potential) appropriate for a dielectric surface, and imposing the 


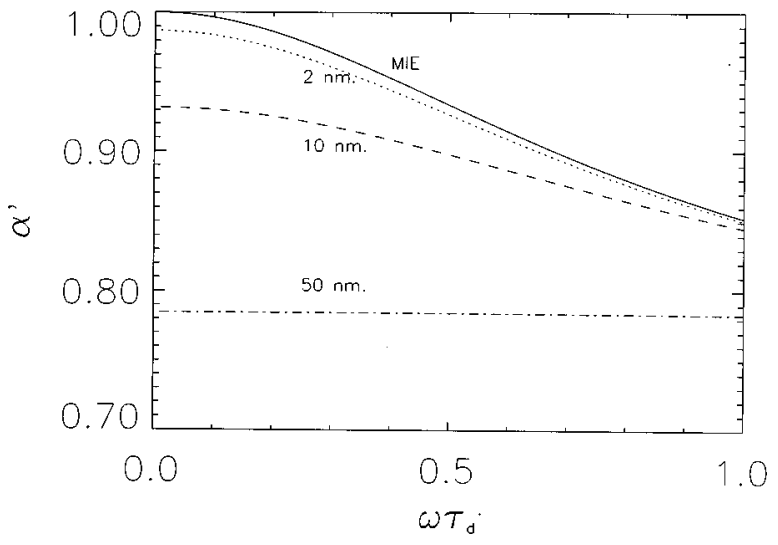

FIG. 1. Frequency dependence of polarizability $\alpha^{\prime}$ for several values of the Debye screening length $L$. The dielectric constant of the sphere is 10 , and the radius is $50 \mathrm{~nm} . \tau_{d}$ is the dielectric relaxation time. The solid line gives the prediction from Mie theory. The deviations from the Mie result are manifestations of spatial dispersion inside the dielectric sphere.

additional requirement that the flow of electrons be tangential at the surface. With $\mathbf{e}_{\perp}$ denoting the normal vector of the surface, tangential flow requires that the following relation holds at the surface:

$$
\mathbf{e}_{\perp} \cdot\left(-\frac{k T}{m_{e}} \boldsymbol{\nabla} n+\frac{e n_{0}}{m_{e}} \mathbf{E}\right)_{\text {surface }}=0 .
$$

For spherical particles, the appropriate solution of the Helmholtz equation is a spherical Bessel function with a complex argument. Solving for the electrostatic potential outside of the sphere with volume $V$ (cf. the Appendix), the induced dipole moment is given by

$$
\mathbf{p}(\omega)=-3 \epsilon_{0} \alpha(\omega) V \mathbf{E}(\omega),
$$

where $\alpha(\omega)=\alpha^{\prime}+i \alpha^{\prime \prime}$ is the complex polarizability of the particle. From elementary electrostatics, two special cases are well known: $\alpha=1$ for a highly conducting sphere (complete screening) and $\alpha=(\epsilon-1) /(\epsilon+2)$ for a nonconducting dielectric sphere. For intermediate conductivities, the polarizability is complex, with a positive imaginary part in order to comply with causality.

The time-averaged power dissipation $W$ in the particle is related to the imaginary part of the polarizability as

$$
W=\frac{3}{2} \epsilon_{0} V \omega \alpha^{\prime \prime} E^{2} .
$$

\section{COMPARISON WITH MIE SCATTERING}

Within the framework of classical electromagnetic theory, a model for the interaction of a small spherical particle with electromagnetic radiation was first worked out by Mie. ${ }^{24,25}$ In this formalism, the solutions of the wave equation do not account for spatial dispersion inside the scatterer. The scattering amplitudes are determined by the complex dielectric constants of the scatterer and the surrounding medium, as well as the geometrical shape of the scattering particle. This problem has been worked out for a variety of geometries. ${ }^{26}$ For a spherical particle with size far smaller than the wave- length of the incoming wave, the polarizability in the Mie approximation is given by

$$
\alpha_{\mathrm{Mie}}=\frac{\epsilon_{d}-1}{\epsilon_{d}+2},
$$

where $\epsilon_{d}$ denotes the complex dielectric constant of a Drude conductor [cf. Eq. (1)]. Note that the Mie approximation to the polarizability does not depend on the particle size. As such, it differs from the exact expression which has a size dependence resulting from spatial dispersion.

Combining with Eq. (12), we find the absorption of a single spherical particle in the Mie approximation,

$$
W_{\text {Mie }}=\frac{3}{2} \epsilon_{0} V \omega \operatorname{Im}\left(\alpha_{\text {Mie }}\right) E^{2} .
$$

The range of validity of the Mie formalism is determined by the conditions under which the effects of spatial dispersion may be neglected. For scattering from a sphere with radius $R$, this implies that Mie theory should be reliable for $|R / \zeta| \gg 1$, where $\zeta$ is the complex Helmholtz length defined in Eq. (7). As discussed in the Appendix, our analytical expression for the polarizability indeed approaches the Mie result in this limit,

$$
\lim _{R / \zeta \rightarrow \infty} \alpha(\omega)=\alpha_{\text {Mie }}(\omega)
$$

With a typical screening length in metals (semiconductors) of $1.0 \AA(10 \mathrm{~nm})$, the Mie treatment loses validity for particle sizes below $\sim 10 \AA(100 \mathrm{~nm})$. As soon as the particle size approaches the screening length, both real and imaginary parts of the polarizability drop substantially below their Mie values.

\section{RESULTS}

The above equations permit evaluation of the dielectric response of the mobile charge carriers to an external harmonic electric field. In particular, they provide a microscopic picture of the electron density and the electron flow inside the particle, and give a model for the complex polarizability of a weakly conducting spherical particle. All calculations are for a semiconductor particle with radius $R=50 \mathrm{~nm}, \epsilon$ $=10$, and placed in vacuo.

Figure 1 shows that the frequency dependence of $\alpha^{\prime}$ is second order. The real part of the polarizability remains essentially unchanged up to $\omega \tau_{d} \approx 0.1$. The dependence of $\alpha^{\prime}$ on the ratio $R / L$ of radius and Debye length is mild, and interpolates smoothly between the two extreme cases of a metallic sphere (complete screening and $\alpha=1$ ) and an isolating dielectric sphere $\left[\alpha=(\epsilon-1) /(\epsilon+2)=\frac{3}{4}\right.$ for $\left.\epsilon=10\right]$. Turning to the dielectric loss factor $\alpha^{\prime \prime}$ (cf. Fig. 2), we note that dielectric loss is much smaller than the prediction from Mie theory as soon as the Debye screening length approaches the particle size. This behavior is not caused by excessive screening, since substantial penetration of the electrical field into the semiconductor particle can still occur. The reason for low dielectric loss is found in the substantial cancellation between the hydrodynamic forces due to the field and due to the electron density gradient. Phrased otherwise, there is substantial cancellation between the last two terms in the Euler equation Eq. (2). In the limit of zero fre- 


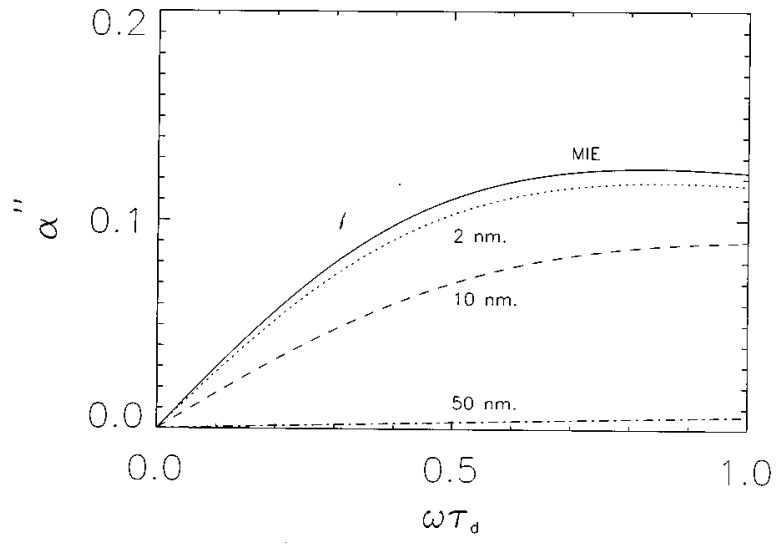

FIG. 2. Frequency dependence of dielectric loss $\alpha^{\prime \prime}$. Curves are labeled as in Fig. 1.

quency, complete cancellation occurs as field-induced drift and density gradient diffusion are equally balanced. The balance is lost for time-dependent fields as the density variations will follow the field with a time lag determined by the dielectric relaxation time $\tau_{d}$. The resulting hydrodynamic flow is dissipative and leads to a nonzero dielectric loss factor $\alpha^{\prime \prime}$. We note that the cancellation is caused by a nonzero electron density gradient. As such, it is a direct manifestation of spatial dispersion and very relevant for situations where the particle size is comparable to the length scale for spatial dispersion (Debye screening length).

Finally, we note that the actual current density carried by the hydrodynamic flow is far smaller than the value expected from the constitutive equation $\mathbf{J}=\sigma \mathbf{E}$, which lies at the basis of the Mie calculation. This is illustrated by the failure of the conventional $^{27,28}$ expression for current-induced power dissipation $W_{c}$,

$$
W_{c}=\frac{1}{2} \sigma \int E^{2}(\mathbf{r}) d V
$$

where the integral is over the volume of the sphere and $\sigma$ is the conductivity. The electric field distribution $E(r)$ is known explicitly from the solution of the wave equation inside the particle (cf. the Appendix), and the volume integral was implemented numerically by a Gaussian integration over

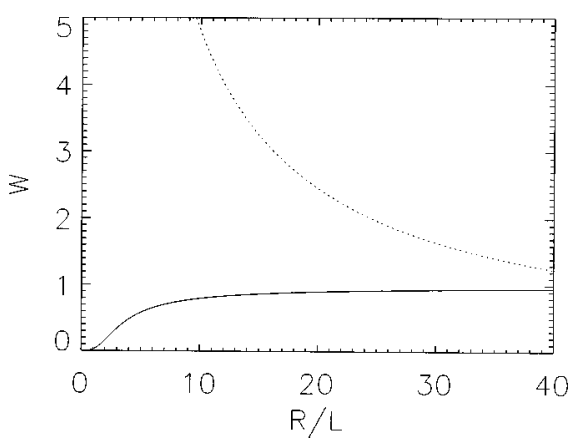

FIG. 3. Power dissipation in a single spherical particle as a function of particle size. The parameters are $\epsilon=10, L=10 \mathrm{~nm}$, and $\omega \tau_{d}=0.1$. The curves are scaled with $W_{\text {Mie }}$, the power loss in the Mie approximation. The solid curve gives the exact result $W / W_{\text {Mie }}$ [cf Eq. (12)], the dashed curve gives the conventional currentinduced power dissipation $W_{c} / W_{\text {Mie }}$ from Eq. (16). the radius of the sphere. A ten-point integration mesh gave adequate numerical accuracy. Figure 3 shows the dependence of the power dissipation on the radius of the sphere. The expression for $W_{c}$ seriously overestimates the power dissipation even for very large particles. We note that the particle size has to exceed the Debye screening length by a factor of at least 40 before the expression Eq. (16) can be considered reasonably reliable.

From Fig. 3 we also note that the Mie approximation gives far better predictions for the power dissipation: It holds for particles with radii exceeding some 20 times the Debye screening length. As the particles get smaller still, the Mie approximation seriously overestimates the dissipated power.

\section{ESTIMATE OF DIELECTRIC CONSTANT OF A POWDER}

Having calculated the complex polarizability of a single weakly conducting sphere, we proceed to estimate the effective dielectric constant of a medium consisting of a collection of such spheres by using the Clausius-Mossotti relation. For this relation to be realistic, we have to assume that the dc conductivity of the powder remains zero, i.e., that the volume fraction of the weakly conducting material remains below the conductivity percolation threshold, ${ }^{29}$ or that spacecharge regions near the semiconductor surface prevent the flow of charge carriers between adjacent spheres. For a monodisperse collection of spheres with volume fraction $f$, the Clausius-Mossotti expression for the complex dielectric constant $\epsilon_{b}=\epsilon_{b}^{\prime}+i \epsilon_{b}^{\prime \prime}$ of the medium is

$$
\frac{\epsilon_{b}-1}{\epsilon_{b}+2}=f \alpha(R) .
$$

As an example, we consider an $n$-doped GaP crystal with a doping density of $5 \times 10^{17} \mathrm{~cm}^{-3}$, which has been made porous by anodic etching in the dark. ${ }^{30}$ In this material the process of anodic etching elegantly leads to geometrical structures with length scales slightly larger than the Debye screening length. As such, it provides a suitable system where effects from spatial dispersion should be important. SEM micrographs revealed a morphology with a porosity of $25 \%$ and a pore wall thicknesses of $\sim 100 \mathrm{~nm}$, suggesting $f$ $=0.75$ and $R=50 \mathrm{~nm}$. Typical parameters are $\epsilon=10$ and $L$ $=10 \mathrm{~nm}$. At $X$-band microwave frequencies we have $\omega \tau_{d}$ $\approx 0.1$ and $\left(\omega / \Omega_{p}\right)^{2} \approx 10^{-6}$. By combining our hydrodynamic electron model with Eq. (17), we estimate an effective dielectric constant of the porous medium $\epsilon_{b}=\left(\epsilon^{\prime}, \epsilon^{\prime \prime}\right)$ $=(7.9,0.42)$, in reasonable agreement with an experimental estimate of $(10 \pm 2,0.5 \pm 0.1) .{ }^{31}$ Note that, in contrast, the Drude model for the dielectric constant [cf. Eq. (1)] predicts an imaginary part of $\epsilon_{d}^{\prime \prime} \approx 100$. We conclude that, even for fairly high doping densities as used here, the dielectric loss in this porous material remains small as a result of spatial dispersion. Turning the argument around, we also conclude that the dielectric loss cannot be interpreted in terms of a simple Drude model or expressions like Eq. (16) as soon as spatial dispersion of the dielectric response is significant. It means that the conventional interpretation of time-resolved microwave conductivity experiments ${ }^{27,32-34}$ on nanostructured semiconductors in terms of charge-carrier concentra- 
tions is seriously compromised if the screening length is comparable to the typical length scale of the nanostructure.

We now address the problem of the relative magnitude and sign of the real and imaginary parts of the dielectric response to an external light stimulus. We can envisage two possibilities. First, the light pulse might increase the density of mobile charge carriers, thereby shortening the Debye screening length. Our model predicts an increase in both $\epsilon_{b}^{\prime}$ and $\epsilon_{b}^{\prime \prime}$, with

$$
\frac{\delta \epsilon_{b}^{\prime} / \delta L}{\delta \epsilon_{b}^{\prime \prime} / \delta L} \approx 3-4 .
$$

As such, the predictions from our model are radically different from the Drude model, and agree qualitatively with experimental observations.

Second, the effect of an intense light pulse may be to increase the volume of the nondepleted region rather than increase its electron density $n_{0}$. For this case, our model again predicts an increase in both $\epsilon_{b}^{\prime}$ and $\epsilon_{b}^{\prime \prime}$ with

$$
\frac{\delta \epsilon_{b}^{\prime} / \delta R}{\delta \epsilon_{b}^{\prime \prime} / \delta R} \approx 5-6
$$

We note that both situations involve an increase in the total number of mobile charge carriers. In contrast, the Mie approximation (no spatial dispersion) fails in both cases. We conclude that the experimentally observed signs and magnitudes of the dielectric response are clear manifestations of the phenomenon of spatial dispersion in the nanostructured network.

\section{DISCUSSION}

The model sketched is based on four simplifying assumptions. First, we assume that the use of the electron density is the sole basic dynamic variable. For static ground-state properties this was legitimized by the work of Hohenberg and $\mathrm{Kohn}^{35}$ and subsequently extended to time-dependent systems also. ${ }^{17,23}$ Second, we assume that the pressure term in the hydrodynamical equations may be approximated by the pressure of a classical ideal gas and that the effects from quantum degeneracy may be neglected. The hydrodynamic approach will fail if the diameter of the semiconductor particle shrinks to the order of the thermal wavelength of the mobile electrons and quantum size effects modify the delocalized electron states involved in charge transport. This limits the validity of our approach to structures larger than several nanometers. Additional quantum effects will arise at high doping densities due to Fermi-Dirac statistics as well as exchange interactions as soon as the thermal wavelength approaches the mean distance between electrons. At room temperature, this implies that the doping density of the semiconductor material should not exceed a value of $\sim 10^{19}$ donors $/ \mathrm{cm}^{3}$. Further deviations from ideality result from long-range Coulomb repulsion between electrons. As these interactions are electrostatic in origin, they tend to be small in semiconductors with high dielectric constant as long as the doping density remains moderate. The contributions from exchange and correlation tend to reduce ${ }^{36}$ the pressure of the electron gas and effectively renormalize the classical
Debye screening length to a shorter value. Third, we assume spherical symmetry for the semiconductor particle and isotropic charge carrier mobility. Straightforward generalization to different shapes (ellipsoids) or anisotropic mobilities leads to anisotropic polarizability tensors for the particle. The calculations are numerically more involved but give comparable qualitative results, provided that the Debye length remains of the order of the particle size. Polydispersity in particle size remains unimportant for low volume fractions. Finally, the fourth strong assumption was that the semiconductor surface plays the double role of dielectric interface as well as confinement boundary for the electrons. This is a highly idealized situation rarely encountered in realistic semiconductor surfaces, where depletion or accumulation layers involve strongly reduced or enhanced electron densities, respectively. These regions are characterized by very strong internal electric fields. ${ }^{37,38}$ For the case of depletion, the confinement boundary lies well below the semiconductor surface and is diffuse rather than well defined. The details of the boundary are known to affect the frequencies of the plasma resonances in the particle. ${ }^{39}$ To describe such situations, the hydrodynamic equations must be linearized around an inhomogeneous unperturbed electron density $n_{0}(\mathbf{r})$, which accounts for the charge-density variation in the depletion or accumulation layer. The boundary conditions at the semiconductor surface should be further modified to account for a surface charge density due to occupied surface states where electrons have been trapped and immobilized. These refinements are conceptually straightforward, but make the algebra too complicated for analytical solution and do not profoundly affect the value of the polarizability of the particle [apart from the fact that the volume $V$ in Eq. (11) stands for the volume of the nondepleted region rather than the volume of the whole particle].

In summary, we have computed the linearized selfconsistent hydrodynamic response of a dilute electron gas inside a small semiconductor particle. It is shown that the effect of conductivity on the static polarizability is very small and interpolates smoothly between the response of a nonconducting dielectric sphere and a perfectly screened metallic sphere. Dielectric screening becomes effective only if the Debye screening length is an order of magnitude smaller than the typical diameter of the semiconductor particle. Even with substantial penetration of the electrical field into the semiconductor, the loss remains small at low frequencies due to very effective cancellation between the electron flows induced by the electric field and by the gradient in the electron density. For realistic situations and moderate doping levels, the dielectric loss of a dispersion of nanocrystallites or a porous semiconductor network is not a reliable estimate of the conductivity of the material as soon as effects from spatial dispersion manifest themselves. In the presence of spatial dispersion, an external light stimulus will increase both the real and imaginary parts of the dielectric constant. The increments will be of comparable magnitude.

\section{APPENDIX: THE COMPLEX POLARIZABILITY OF A WEAKLY CONDUCTING SPHERE}

At low frequencies, the particle size is orders of magnitude below the wavelength of the electrical field $\mathbf{E}$. The field 
therefore may be considered homogeneous on the scale of the particle. We assume that the electric field is directed along the $z$ axis. For this case, the electrostatic potential outside of the sphere has the form (in spherical coordinates)

$$
\Phi_{o}(r, \theta)=\left(-E r+\frac{1}{4 \pi \epsilon_{0}} \frac{p}{r^{2}}\right) P_{1}(\cos \theta),
$$

where the second term is the polarization field caused by the dipole moment $p$ induced in the sphere. In view of rotational symmetry of the Helmholtz equation, the potential inside the sphere must have a pure $P_{1}(\cos \theta)$ angular dependence as well, as other spherical harmonics are not necessary to satisfy the boundary conditions. For the radial dependence, the fourth-order Helmholtz differential equation has four independent solutions, two of which are regular at the origin of the sphere. Taking the linear combination of these two regular solutions, the potential inside the sphere has the form

$$
\Phi_{i}(r, \theta)=\left[a B\left(\frac{r}{\zeta}\right)+b r\right] P_{1}(\cos \theta) .
$$

The solution of the Helmholtz equation $B$ is defined in terms of the modified spherical Bessel functions of the first kind,

$$
B(z)=3 \zeta\left(\frac{\pi}{2 z}\right)^{1 / 2} I_{3 / 2}(z) .
$$

The normalization was chosen such that it reduces to the solution of the Poisson equation in the limit of large Debye length,

$$
\lim _{\zeta \rightarrow \infty} B\left(\frac{r}{\zeta}\right)=r .
$$

The three unknowns $a, b$, and $p$ are determined from the three boundary conditions at the surface of the sphere:

(i) $\Phi_{i}(R)=\Phi_{0}(R)$,

(ii) $\epsilon(\partial / \partial r) \Phi_{i}(R)=(\partial / \partial r) \Phi_{0}(R)$,

(iii) $\partial /\left.\partial r\left(\Phi_{i}-L^{2} \Delta \Phi_{i}\right)\right|_{r=R}=0$,

where $\epsilon$ is the (real) dielectric constant of the dielectric background in which the electrons move. The third condition imposes the physical requirement that the electron flow be tangential at the surface of the sphere.

From straightforward but tedious algebra, the magnitude of the dipole moment $p$ may be solved in closed form:

$$
p(\omega)=\frac{4}{3} \pi R^{3} 3 \epsilon_{0} \alpha E,
$$

where the complex polarizability $\alpha$ of the sphere is given by

$$
\begin{gathered}
\alpha=\frac{[\epsilon-\delta(\epsilon-1)] \frac{R}{\zeta} B^{\prime}-B}{[\epsilon-\delta(\epsilon+2)] \frac{R}{\zeta} B^{\prime}+2 B} \\
\delta=\left(\frac{\omega}{\Omega_{p}}\right)^{2}+i \omega \tau_{d} .
\end{gathered}
$$

The validity of this analytical expression has been verified for three special cases:

(i) For a nonconducting sphere,

$$
\lim _{L \rightarrow \infty} \alpha=\frac{\epsilon-1}{\epsilon+2} .
$$

(ii) For a highly conducting (metallic) sphere,

$$
\lim _{L \downarrow 0} \alpha=1 .
$$

(iii) The limit of large particles reproduces the result from the Mie formalism,

$$
\lim _{R / \zeta \rightarrow \infty} \alpha(\omega)=\frac{(1-\delta) \epsilon+\delta}{(1-\delta) \epsilon-2 \delta}=\frac{\epsilon_{d}(\omega)-1}{\epsilon_{d}(\omega)+2},
$$

where $\epsilon_{d}(\omega)$ stands for the dielectric constant of a Drude conductor [cf. Eq. (1)].

It is of interest to consider the static polarizability of the sphere as well. In the limit of zero frequency, the complex Helmholtz length $\zeta$ approaches the Debye screening length

$$
\lim _{\omega \downarrow 0} \zeta=L
$$

and the polarizability becomes real. As a function of $L$, the polarizability $\alpha$ interpolates smoothly between the nonconducting and metallic limit,

$$
\frac{\epsilon-1}{\epsilon+2}<\alpha(\omega=0, L)<1 .
$$

As a concluding remark, we note that the self-consistent potential inside the sphere is such that the electrical field is neither homogeneous nor directed along the $z$ axis. In this, the conducting case is characteristically different from the nonconducting dielectric case.
${ }^{1}$ A. G. Cullis and L. T. Canham, Nature (London) 353, 335 (1991).

${ }^{2}$ C. Delerue, G. Allan, and M. Lannoo, Phys. Rev. B 48, 11024 (1993).

${ }^{3} \mathrm{cf}$. the special section in Science 254, 1300 (1991).

${ }^{4}$ Dielectric Properties of Heterogeneous Materials, edited by A. Priou (Elsevier, New York, 1992).

${ }^{5}$ A. Henglein, Chem. Rev. 89, 1861 (1989).

${ }^{6}$ L. Brus, Appl. Phys. A: Solids Surf. 53, 465 (1991).

${ }^{7}$ A. Read, R. Needs, J. Nash, L. Canham, P. Calcott, and A. Otesh, Phys. Rev. Lett. 69, 1232 (1992).
${ }^{8}$ B. K. Ridley, Quantum Processes in Semiconductors (Clarendon, Oxford, 1993).

${ }^{9}$ H. Weller, Adv. Mater. 5, 88 (1993).

${ }^{10}$ M. Lannoo, C. Delerue, and G. Allan, Phys. Rev. Lett. 74, 3415 (1995).

${ }^{11}$ A. Hagfeldt and M. Grätzel, Chem. Rev. 95, 49 (1995).

${ }^{12}$ B. H. Erné, D. Vanmaekelbergh, and J. Kelly, Adv. Mater. 7, 739 (1995).

${ }^{13}$ J. Orton and M. Powell, Rep. Prog. Phys. 43, 81 (1980).

${ }^{14}$ Transport, Correlation and Structure Defects, edited by $\mathrm{H}$. 
Fritzsche (World Scientific, Singapore, 1990).

${ }^{15}$ A. Henglein, Top. Curr. Chem. 143, 113 (1988).

${ }^{16}$ J. Perenboom, P. Wyder, and F. Meier, Phys. Rep. 78, 173 (1981)

${ }^{17}$ R. Parr and Weitao Yang, Density Functional Theory of Atoms and Molecules (Oxford University Press, New York, 1989).

${ }^{18}$ F. Bloch, Z. Phys. 81, 363 (1933).

${ }^{19}$ See, e.g., B. Deb and S. Ghosh, The Single Particle Density in Physics and Chemistry, edited by N. March and B. Deb (Academic, London, 1987), Chap. 8.

${ }^{20}$ V. Kresin, Phys. Rep. 220, 1 (1992).

${ }^{21}$ M. Brack, Rev. Mod. Phys. 65, 677 (1993).

${ }^{22}$ L. Féret et al., J. Phys. B 29, 4477 (1996).

${ }^{23}$ A. Domps, P. Reinhard, and E. Suraud, Phys. Rev. Lett. 80, 5520 (1998).

${ }^{24}$ G. Mie, Ann. Phys. (Leipzig) 25, 377 (1908).

${ }^{25}$ See, e.g., H. van de Hulst, Light Scattering by Small Particles (Wiley, New York, 1957).

${ }^{26}$ A. P. van Gelder, J. Holvast, J. Stoelinga, and P. Wyder, J. Phys. C 4, 2757 (1972).

${ }^{27}$ M. de Haas, Ph.D. thesis, Delft University, 1977.

${ }^{28}$ H. M. Altschuler, in Handbook of Microwave Measurements, edited by M. Sucher and J. Fox (Wiley, New York, 1963), Vol. II.
${ }^{29}$ D. Bergman and D. Stroud, in Solid State Physics, edited by H. Ehrenreich and D. Turnbull (Academic, Boston, 1992), Vol. 46.

${ }^{30}$ B. Erne, Ph.D. thesis, Utrecht University, 1995.

${ }^{31} \mathrm{~W}$. Lubberhuizen (private communication). The determination was done by loading an $X$-band microwave cavity with a small piece of porous $\mathrm{GaP}$ and analyzing the effect on the resonance frequency and width.

${ }^{32}$ J. Warman, M. de Haas, M. Grätzel, and P. Infelta, Nature (London) 310, 306 (1984).

${ }^{33}$ K. Schindler and M. Kunst, J. Phys. Chem. 94, 822 (1990).

${ }^{34}$ S. Martin, H. Herrmann, and M. Hoffmann, J. Chem. Soc., Faraday Trans. 90, 3315 (1994); 90, 3323 (1994).

${ }^{35}$ P. Hohenberg and W. Kohn, Phys. Rev. 136, B864 (1964).

${ }^{36}$ For the nondegenerate electron gas, the effects of correlation, exchange, and Coulombic interaction are discussed in A. Fetter and J. Walecka, Quantum Theory of Many Particle Systems (McGraw-Hill, New York, 1971), Chap. 8.

${ }^{37}$ H. K. Henisch, Semiconductor Contacts (Clarendon, Oxford, 1985).

${ }^{38}$ S. R. Morrison, The Chemical Physics of Surfaces, 2nd ed, (Plenum, New York, 1990).

${ }^{39}$ R. Ruppin, J. Opt. Soc. Am. 66, 449 (1976). 